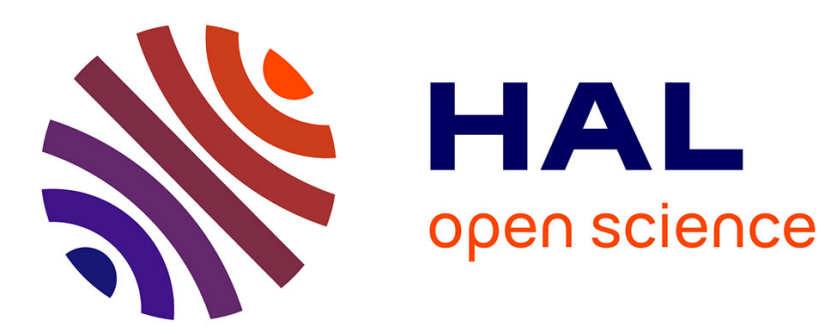

\title{
Collocation, phrasème, dénomination: vers une théorie de la créativité phraséologique
}

Christopher Gledhill, Pierre Frath

\section{To cite this version:}

Christopher Gledhill, Pierre Frath. Collocation, phrasème, dénomination: vers une théorie de la créativité phraséologique. La Linguistique, 2007, 43 (1), pp.65-90. 10.3917/ling.431.0063 . hal01220295

\section{HAL Id: hal-01220295 \\ https://hal-univ-paris.archives-ouvertes.fr/hal-01220295}

Submitted on 28 Jun 2021

HAL is a multi-disciplinary open access archive for the deposit and dissemination of scientific research documents, whether they are published or not. The documents may come from teaching and research institutions in France or abroad, or from public or private research centers.
L'archive ouverte pluridisciplinaire HAL, est destinée au dépôt et à la diffusion de documents scientifiques de niveau recherche, publiés ou non, émanant des établissements d'enseignement et de recherche français ou étrangers, des laboratoires publics ou privés. 
Gledhill, Christopher \& Pierre Frath. 2007. Collocation, phrasème, dénomination : vers une théorie de la créativité phraséologique. La Linguistique 43(1). 65-90. ISBN 978-2-13-0562641. (PDF)

\title{
COLLOCATION, PHRASEME, DENOMINATION: VERS UNE THEORIE DE LA CREATIVITE PHRASEOLOGIQUE
}

\author{
par Christopher GLEDHILL \\ Université Marc Bloch, Strasbourg \\ Pierre Frath \\ Université de Reims Champagne-Ardenne
}

\begin{abstract}
In this article, we address the question of phraseological innovation. By this, we refer to any new expression which does not constitute a regular collocation (a known expression) within a given language. Our objective is not to propose a theory of stylistic creativity. Instead, we limit our investigation to explaining how an expression comes to be integrated in the lexicogrammar as defined by M.A.K. Halliday. We also discuss various issues of terminology, notably the oppositions collocation / colligation, synthème / phrasème which have currently come to the fore in the fields of lexicography and French functional linguistics. These terms do not in fact help us to deal with the problem of phraseological innovation. So we turn instead to the semiotic notion of denomination, defined as the potential for a sign to refer globally within a given discourse. This approach avoids the traditional debate about whether expressions are syntactically fixed or semantically opaque. Rather, the main defining criterion which is of interest to us is the moment when a construction becomes independent of the discourse in which it is formulated in order to be assimilated as an expression by the speech community. As a case in point, we examine the emergence of a new expression in an extract from Trainspotting by Irine Welsh.
\end{abstract}

Selon une conception très répandue, seule une grammaire abstraite de la pensée pourrait expliquer notre capacité à encoder et décoder des idées nouvelles. Mais l'importance de «l'encodage » dans l'innovation linguistique est difficile à justifier si l'on considère, comme les sociolinguistes, que la valeur et même l'existence de toute construction nouvelle est le fruit d'une négociation au sein de la communauté linguistique. On parle ainsi de l'acception d'un mot ou d'une expression plutôt que de son sens inhérent. Plus récemment, la linguistique empirique (la psycholinguistique et la linguistique de corpus) a souligné le rôle des expressions conventionnelles dans le discours. L'innovation, selon ces linguistes, serait le résultat d'un contraste avec une toile de fond phraséologique pré-existante plutôt que la traduction d'une idée nouvelle en langue naturelle. Mais si ces approches ont miné le concept de la génération et de la transmission de l'information, elles n'apportent toutefois pas de réponse à des questions essentielles : comment identifier une expression nouvelle et comment expliquer son interprétation dans le cadre d'une théorie linguistique?

Dans cet article nous abordons la question de la créativité phraséologique. Nous désignons par ce terme toute innovation expressive qui ne constitue pas une collocation régulière (une expression déjà connue) dans une langue donnée. Notre objectif n'est pas d'avancer une théorie de l'innovation stylistique. Nous nous proposons plutôt d'examiner comment une expression inédite peut s'intégrer dans le système lexico-grammatical tel qu'il est défini par Michael Halliday ${ }^{1}$. Nous donnons également quelques précisions quant à la terminologie dans ce domaine, notamment les oppositions collocation / colligation, synthème / phrasème qui ont récemment pris de l'importance dans des domaines de la lexicographie et de la linguistique

1. Michael Halliday \& Christian Matthiessen, 2004, [1 $1^{\text {ère }}$ éd. 1985], Introduction to Functional Grammar. Londres, Arnold. 
fonctionnelle. Toutefois, ces oppositions ne suffisent pas à expliquer les phénomènes qui nous intéressent ici. Nous nous tournons donc vers la notion sémiotique de dénomination, c'est-àdire la capacité d'un signe à réaliser une référence globale dans un discours. Cette approche a l'avantage d'éviter toute décision concernant le figement syntaxique ou l'opacité sémantique d'une unité phraséologique. Le critère définitoire pour l'innovation phraséologique consiste alors plutôt à identifier le moment où une construction devient indépendante du discours dans laquelle elle a été formulée pour être assimilée en tant qu'expression par la communauté linguistique.

Pour démontrer notre approche, nous nous bornerons à étudier une construction résultative en anglais écossais. Il s'agit d'expliquer comment ce signe a pris sa place dans la littérature britannique contemporaine. Mais avant d'aborder cette analyse, il convient de passer en revue la notion de créativité phraséologique, au moins telle qu'elle est conçue chez certains linguistes descriptivistes et fonctionnalistes.

\section{COLLOCATION ET COLLIGATION}

Les linguistes empiriques appartenant à la tradition de Firth affirment que les locuteurs puisent dans leur mémoire des expressions relativement stables au fur et à mesure de l'énonciation. Le terme le plus souvent employé pour ces ensembles est collocation, un terme qui comprend des syntagmes réguliers (poser une question, une pluie diluvienne) ainsi que des locutions plus figées (au fur et à mesure, chemin de fer). Ces dernières correspondent, comme on le verra plus loin, aux synthèmes de Martinet. Si la plupart des linguistes s'accordent sur la nature relativement figée de ces expressions ${ }^{2}$, ils omettent souvent de souligner que la collocation ne réfère ni au figement de l'unité, ni à la séquence lexicale. La collocation est plutôt un principe de cooccurrence lexicale qui a d'abord été formulée par Firth en termes d'attraction mutuelle sans prendre en compte l'ordre des mots ni leur proximité :

«Collocations of a given word are statements of the habitual or customary places of that word in collocational order but not in other contextual order and emphatically not in any grammatical order. The collocation of a word or a 'piece' is not to be regarded as mere juxtaposition, it is an order of mutual expectancy. ${ }^{3}$

De même, quand il s'agit d'identifier une relation mutuelle entre des catégories grammaticales, Firth avait employé le terme de colligation. Il oppose ainsi la collocation d'occurrences push + through à la colligation de classes VERBE + PARTICULE. Cette notion a souvent été assimilée à la collocation grammaticale. Le terme est parfois aussi utilisé par certains analystes de corpus ${ }^{4}$ pour désigner la tendance de certains mots ou constructions à occuper une place précise dans la chaîne parlée.

Mais si l'opposition collocation / colligation a connu un certain succès dans la lexicologie et l'analyse de corpus, elle présente néanmoins un inconvénient théorique particulier. En postulant que tel ou tel ensemble de mots est une collocation (ou une colligation) les linguistes descriptivistes supposent que les éléments dont est composée l'expression possèdent des propriétés indépendantes qui causent une relation mutuelle ou en sont à l'origine. Or, nous avons argumenté ailleurs ${ }^{5}$ que «l'attraction mutuelle » ne constitue pas une explication satisfaisante des phénomènes observés, et que ce que nous avions appelé une "colle » syntaxique ou sémantique ne peut exister sans postuler une série d'entités compositionnelles,

2. Gaston Gross, 1996, Les Expressions figées, Paris, Ophrys.

3. John R. Firth, 1957, A Synopsis of Linguistic Theory. 1930-1955, Oxford, OUP, p.12

4. Michael Hoey, 2005, Lexical Priming : A New Theory of Words and Language, London, Routledge.

5. Christopher Gledhill \& Pierre Frath, 2005, «Free-Range Clusters or Frozen Chunks? Reference as a Defining Criterion for Linguistic Units », Recherches anglaises et Nord-américaines, Strasbourg, Vol. 38, p.2543. 
qualia, opérateurs, fonctions lexicales, etc. qui seraient, selon nous, des spéculations métaphysiques impossibles à justifier et essentiellement ad hoc. Nous proposerons une alternative à cette impasse en fin d'article.

Pour l'instant, il serait approprié de remplacer l'opposition collocation / colligation par la notion plus synthétique de lexico-grammaire développée par Michael Halliday ${ }^{6}$. Son principe est simple : chaque forme lexicale a une lexico-grammaire spécifique, et de même, chaque construction lexico-grammaticale est associée à une série restreinte de formes lexicales. Ainsi une complétive nominale comme the belief that the world is round «la conviction que la terre est ronde » dépend-elle en anglais et en français de l'existence d'un certain nombre de lexèmes appartenant à un champ sémantique restreint, les projections nominales : fait, conviction, idée. Ces constructions existent seulement parce qu'elles constituent des expressions sur le plan sémiotique; en l'occurrence une façon conventionnelle de commenter des procès cognitifs (établis dans la complétive) et de les mettre en relation avec l'argumentation suivante (dans le prédicat). De même, les résultatives de déplacement du type, She laughed her way through the crowd «Elle s'est frayé un chemin en riant à travers la foule » nécessitent en anglais le complément de déplacement way et un complément de direction through the crowd. La grammaire traditionnelle considère qu'on a ici une mise en relation d'une résultative, to $\mathrm{X}$ one's way though $\mathrm{Y}$, et deux référents her et the crowd. Mais on peut voir que ces trois éléments, y compris la résultative, ne sont pas des variables libres, mais des lexèmes dont la mise en place est totalement régie par la phraséologie conventionnelle de l'anglais : the crowd fait partie, par convention, des participants à travers lesquels on veut passer. De même, l'emploi «atypique » du verbe laugh s'inscrit aussi dans la lexico-grammaire de l'anglais : la résultative en way permet cet emploi, parce que la fonction primordiale de cette construction est d'exprimer un mouvement paradoxal.

Ces correspondances ne sont pas le résultat d'une attraction mutuelle entre mots et catégories. Elles incarnent plutôt une relation étroite entre pensée et expression, deux facettes inséparables d'un seul système. L'originalité de cette approche est de postuler que la lexicogrammaire remplit deux rôles à la fois : elle sert d'interface par rapport au contexte sociosémantique en même temps que de matrice pour l'organisation de la parole. Selon Halliday :

«We use language to make sense of our experience, and to carry out our interactions with other people. This means that the grammar has to interface with what goes on outside language: with the happenings and conditions of the world, and with the social processes we engage in. But at the same time it has to organize the construal of experience, and the enactment of social processes, so that they can be transformed into wording. ». ${ }^{7}$

L'absence de distinction entre pensée et parole pose problème pour la grammaire formelle qui postule des transformations ou autres opérations sur des préconstruits primitifs de la pensée abstraite. Au contraire, la lexico-grammaire conçoit notre compétence communicative en termes de cascades de choix : un choix sémiotique, une expression, est réalisé par une série de composants grammaticaux et lexicaux, une construction, et vice versa. Nous verrons plus loin comment ce principe détermine notre façon de voir les résultatives en anglais.

Cette conception de la lexico-grammaire n'est pas très répandue en dehors des travaux grammaticaux inspirés par Halliday, par exemple Hunston et Francis ${ }^{8}$. Dans le domaine de la

6. Michael Halliday, 1966, « Lexis as a Linguistic Level », Bazell, C., Catford, J. Halliday, M.A.K. \& Robins, R. In Memory of J. R. Firth, London, Longmans, p.148-162.

7. Michael Halliday \& Christian Matthiessen, 2004, [1ère éd. 1985], Introduction to Functional Grammar. Londres, Arnold, p. 24-25.

8. Susan Hunston et Gill Francis, 2000, Pattern Grammar - A Corpus-Driven Approach to the Lexical Grammar of English, Amsterdam, John Benjamins. 
lexicologie, on peut toujours rencontrer, par exemple dans Nesselhauf ${ }^{9}$, la distinction entre collocation et combinaison libre. Selon Nesselhauf, un verbe fréquent comme want entre en relation avec un nombre de compléments plus varié qu'un verbe moins fréquent, comme require, et il s'ensuit, selon elle, que les compléments de want sont des combinaisons libres par rapport aux collocations restreintes de require. Mais il est possible de démontrer que les deux verbes ont une distribution asymétrique de collocations : a wanted man " un homme recherché »/ the required material «le matériel nécessaire », ainsi qu'une distribution asymétrique de colligations : I want to have a baby / *I require to have a baby, This wants fixing / This requires fixing, *I want that you fix it / I require that you fix it, etc. Force est de constater que la fréquence et la diversité des emplois ne suffisent pas pour démontrer que deux formes constituent une combinaison libre. Nous croyons plutôt que tout lexème fait partie d'un faisceau plus ou moins restreint de constructions lexico-grammaticales. Or, nous avons démontré, dans l'étude d'un corpus spécialisé ${ }^{10}$, que même des mots grammaticaux possèdent des restrictions lexico-grammaticales de ce genre.

Mais si nous acceptons que chaque mot ou lexème possède des relations lexicogrammaticales, comment expliquer que les locuteurs puissent être créatifs en juxtaposant des éléments linguistiques dont les formes ne sont pas complètement libres? Une réponse a été formulée, entre autres, par John Sinclair. Son principe idiomatique suppose que notre production linguistique consiste en une série de choix pré-construits. Pour expliquer l'existence de formulations nouvelles, Sinclair postule un choix ouvert, une dimension dans laquelle chaque choix lexical mène à une série restreinte de nouvelles constructions grammaticales :

«This is a way of seeing language text as the result of a very large number of complex choices. At each point where a unit is completed (a word, a phrase or clause), a large range of choice opens up and the only constraint is grammaticalness. ${ }^{11}$

Selon Sinclair, les deux principes opèrent en parallèle, mais sont essentiellement contradictoires :

«For normal texts we can put forward the proposal that the first mode to be applied is the idiom principle [...] The open-choice analysis could be imagined as an analytical process which goes on in principle all the time, but whose results are only intermittently called for. $»^{12}$

Cependant, dans une perspective lexico-grammaticale, il est évident que les contraintes de grammaticalité sur les choix ouverts sont les mêmes que celles qui régissent le principe idiomatique. Il n'existe donc pas de choix véritablement ouvert, et on est obligé de constater que tout mot entre dans des relations lexico-grammaticales, sans exception.

En affirmant qu'une nouvelle expression correspond tout simplement à un nouvel agglomérat lexical, Sinclair soutient implicitement que la portée d'une expression est le résultat mécanique de la conjonction de deux ou plusieurs vocables. L'énoncé ne serait que l'encodage d'une pensée symbolique, un processus cognitif qui se décrit en termes de règles. Or, les énoncés simples, au fur et à mesure, ou complexes, She laughed her way through the crowd, n'encodent rien. Les signes sont les constituants même de la pensée, et ils ne sauraient être exprimés facilement autrement. Ils peuvent d'ailleurs faire l'objet de définitions assez

9. Nadja Nesselhauf, 2003, «The Use of Collocations by Advanced Learners of English and Some Implications for Teaching », Applied Linguistics, Vol. 42/2, p.223-242.

10. Christopher Gledhill, 2000, Collocations in Science Writing, Tübingen, Gunter Narr Verlag.

11. John Sinclair, 1991, Corpus, Concordance, Collocation, Oxford, Oxford University Press, p.109.

12. John Sinclair, 1991, .., p.114. 
longues dans les dictionnaires. On pourrait même affirmer que ces deux constructions correspondent à des expressions idiomatiques, selon la définition de Cruse :

\footnotetext{
«We shall require two things of an idiom : first that it be lexically complex - i.e. it should consist of more than one lexical constituant; second that it should be a single minimal semantic constituent. »13
}

Pour l'instant, nous sommes tenus de conclure qu'il n'existe pas deux systèmes comme on le pense souvent : l'un idiomatique quasi-autonome où la production et l'interprétation sont instantanées, et l'autre ouvert, régi par des règles de la combinatoire grammaticale traditionnelle. Au contraire, nous devons supposer que tout ce que nous formulons dans notre production langagière est conforme aux contraintes de la lexico-grammaire, un système qui constitue l'interface inséparable entre la parole et la signification.

\section{SYNTHEME ET PHRASEME}

Les linguistes de corpus descriptivistes ${ }^{14}$, supposent que les ensembles faire la sieste et chemin de fer sont des collocations parce que le choix d'un de leurs éléments lexicaux permet de prédire la présence des autres : il s'agit d'un constat de probabilité basé sur l'observation. Mais comme nous l'avons vu, l'approche lexico-grammaticale suppose aussi que ces ensembles sont des constructions parce que leurs composants obéissent aux contraintes du système lexico-grammatical. Par contraste, le terme expression s'applique essentiellement à l'existence de ces ensembles en tant que signes. Certaines expressions n'ont pas besoin de détermination dans un contexte donné pour qu'on les reconnaisse, tels les lexèmes chemin de fer, et au fur et à mesure. Pourtant, l'approche lexico-grammaticale a plus de mal à concevoir l'existence indépendante de constructions moins figées comme poser une question, to laugh one's way through the crowd, car elles ont besoin d'être réalisées en contexte avant qu'on reconnaisse leur fonction expressive. La question est de savoir s'il est possible, ou même désirable, de postuler l'existence de signes de ce type, situés entre le mot et la phrase?

C'est justement cette tension entre l'existence virtuelle d'une expression et sa réalisation qui est abordée par André Martinet ${ }^{15}$. Dans l'approche fonctionnaliste sur les expressions figées, le terme synthème est employé pour désigner des unités qui sont le résultat de processus de dérivation, de composition (chemin de fer) ou de figement syntagmatique incomplet (faire la sieste). Selon sa définition, le synthème est une unité linguistique qui fonctionne syntaxiquement comme un monème complexe :

«...il s'agit d'une unité linguistique signifiante, désignant une notion bien définie, mais où la forme permet de distinguer des éléments successifs porteurs au départ de sens distinctifs. ${ }^{16}$

L'unité du synthème dépend spécifiquement de sa fonction syntaxique. Les critères de sa définition sont ainsi:

«... a) l'impossibilité de déterminer individuellement les monèmes constituants, b) l'obligation de tout synthème de s'intégrer dans une classe préétablie de monèmes. ${ }^{17}$

13. David Cruse, 1986, Lexical Semantics, Cambridge, Cambridge University Press, p.37

14. Nous ne citons que trois études récentes dans ce domaine : Michael Stubbs, 2001, Words and Phrases, Corpus Studies of Lexical Semantics, London, Blackwell, Francis Grossmann et Agnès Tutin, 2003, Les collocations - analyse et traitement, Amsterdam, De Werelt, Stefan Evert, 2005, The Statistics of Word Cooccurrences : Word Pairs and Collocations, Thèse doctorale, Institut für maschinelle Sprachverarbeitung, Université de Stuttgart.

15. André Martinet, 1979, Grammaire fonctionnelle du français, Paris, Credif, p. 250

16. André Martinet, 1999, «Le synthème », La Linguistique, Vol 35/2, p. 11.

17. André Martinet, $1999, \ldots$, p. 15. 
Cette restriction cerne de façon assez nette les lexicalisations chemin de fer, une locution nominale, et faire la sieste, une locution verbale. La notion de synthème est ainsi, par rapport à la collocation, plus discriminatoire et plus dynamique dans la mesure où l'on met l'accent sur l'origine discursive - et donc étymologique - de l'ensemble. Cette perspective a été reprise par Depecker, qui suggère le terme phrasème pour des constructions plus complexes. Le phrasème est :

«...une unité supérieure au synthème en raison du jeu possible de ses éléments pris entre la dynamique de la phrase et le complexe sémantique ainsi constitué... autant ce genre d'unité peut évoluer vers un synthème (image du satellite $=>$ image satellite), autant il peut être libéré dans la phrase à partir d'un synthème (image satellite $=>$ image transmise par le satellite). ${ }^{18}$

La notion de 'jeu' est cruciale pour cette définition, car elle permet notamment de concevoir le système phraséologique comme un système de reformulations entre expressions apparentées. La reformulation peut correspondre à la réduction dans le cas d'un monème comme métro (à partir du synthème chemin de fer métropolitain), ou à l'expansion pour un phrasème comme elle a fait une très bonne sieste (apparenté à faire une sieste / faire la sieste). Les énoncés complets tels que les projections ou les résultatives que nous avons évoquées plus haut seraient donc des phrasèmes. Ce qui est significatif dans l'approche de Depecker est qu'il souligne l'unité sémantique non seulement des monèmes et synthèmes, mais aussi des phrasèmes, la seule différence étant le potentiel de variation des éléments qui les composent.

Nous sommes donc très loin d'une vision probabiliste adoptée par les linguistes de corpus, notamment Sinclair, qui ne discernent aucune hiérarchie parmi les collocations et aucune relation entre des reformulations apparentées. Mais si nous utilisons l'étiquette phrasème pour décrire la résultative She laughed her way through the crowd, dans quelle mesure peut-on dire que cette désignation explique l'originalité ou même le fonctionnement de cette expression dans un discours donné? Et dans quelle mesure est-il possible ou même nécessaire d'identifier les éléments «originels » des synthèmes ou des phrasèmes? Et dans le sens inverse, est-il possible d'établir le parcours discursif de l'expression elle a fait une bonne sieste, à partir de faire la sieste ou faire une sieste? D'ailleurs, pourquoi insister sur des termes nouveaux lorsque l'on dispose de termes traditionnels avec des portées comparables : locution (=> synthème), groupe / syntagme / phrase (=> phrasème)?

Si ces considérations ne répondent pas directement à notre question de départ, elles nous permettent néanmoins de la reposer de façon plus précise. Nous avons postulé plus haut que toute forme lexicale (mot, morphème ou monème, selon les théories) possède des relations de cooccurrence qui ne sont pas les propriétés du mot individuel, mais font plutôt partie d'un système global, la lexico-grammaire. C'est d'ailleurs cette appartenance à un système qui définit, à notre avis, la notion de lexème. Ce que l'opposition synthème / phrasème ajoute à notre débat est un moyen commode de classer des unités phraséologiques par leur origine et leur parenté à d'autres formulations. Une innovation phraséologique peut donc être définie comme une collocation tout à fait nouvelle (une cooccurrence lexicale unique) qui peut être située quelque part dans le continuum de réalisation monème (ou morphème) - synthème phrasème. La distinction synthème / phrasème permet de constater les différents degrés de détermination de certaines constructions. Mais nous verrons plus loin que même cette distinction n'est pas suffisante. Selon la terminologie que nous proposons ici, une nouvelle

18. Loïc Depecker, 1999, « Monème, synthème et phrasème. Essai d'introduction du concept de phrasème dans la théorie fonctionnaliste », La Linguistique, Vol 35/2, p.43. 
construction doit impérativement être assimilée dans le discours et doit produire de nouvelles variations avant de devenir une expression. Nous décrirons plus loin l'importance de ce processus sous l'appellation de dénomination.

\section{LE CAS D'UNE RESULTATIVE DANS TRAINSPOTTING}

L'exemple que nous avons choisi d'examiner ici provient du roman écossais Trainspotting, un texte emblématique, devenu célèbre et souvent cité en Grande-Bretagne dans les années 1990. Notre phrase comporte des expressions typiquement écossaises, et notamment une résultative qui a eu un grand succès et est devenue très célèbre : The most wretched, servile, miserable, pathetic trash that was ever shat intae creation. La construction résultative est réputée être très productive en anglais moderne, à tel point qu'elle est devenue l'exemple type des travaux de Goldberg (dont nous discuterons plus loin). Notre méthode consiste à confronter cette formule à la phraséologie ambiante de la langue anglaise, telle qu'elle apparaît dans un corpus électronique de l'anglais britannique de cette époque : le British National Corpus (BNC). Nous examinerons l'ensemble de cette phrase, constituée d'une comparaison discontinue, The most ... that was ever, d'une énumération d'épithètes, wretched, servile, miserable, pathetic, d'un passif that was ever shat et d'un groupe prépositionnel intae (=into) creation.

Pour examiner le style de l'auteur, Irvine Welsh, nous aurions pu comparer Trainspotting à un corpus de textes écossais, un corpus littéraire, ou aux œuvres dont Welsh lui-même se serait inspiré. Mais il est aussi intéressant d'observer à quel point la phraséologie d'une oeuvre peut puiser dans le discours collectif pour enfin l'intégrer à nouveau. Le BNC est un corpus de textes d'origine britannique de la fin des années 1980, avec des passages d'auteurs canoniques de l'époque (par exemple David Lodge) et des transcriptions de la langue parlée (environ $10 \%$ du corpus).

A première vue, l'originalité de notre exemple repose entièrement sur le choc et la surprise suscités par le verbe shat (le prétérit de to shit) pour exprimer la création de la nation écossaise. La phrase est employée à la fin d'un paragraphe d'invective, écrit en eye-dialect, c'est-à-dire une orthographe littéraire qui évoque l'accent écossais, sans le représenter phonétiquement :

«Fuckin failures in a country of failures. It's nae good blamin it oan the English for colonising us. Ah don't hate the English. They're just wankers. We are colonised by wankers. We can't even pick a decent, vibrant, healthy culture to be colonised by. No. We're ruled by effete arseholes. What does that make us? The lowest of the fuckin low, the scum of the earth. The most wretched, servile, miserable, pathetic trash that was ever shat intae creation. Ah don't hate the English. They just git oan wi the shite thuv goat. Ah hate the Scots. ${ }^{19}$

«Foutus ratés dans un pays de ratés. C'est inutile de reprocher aux Anglais de nous avoir colonisés. Je ne hais pas les Anglais. Ce ne sont que des branleurs. Nous sommes colonisés par des branleurs. On n'a même pas été capables de trouver une civilisation saine, radieuse, honnête pour nous envahir. Pas du tout. Nous sommes gouvernés par des trous du cul décadents. Et qu'est-ce que ça fait de nous? Les plus minables des minables, la lie de la terre. Les plus misérables, les plus serviles, les plus lamentables, les plus pathétiques déchets que la Création ait jamais produits. Je ne hais pas les Anglais. Ils font ce qu'ils peuvent avec leur propre merde. Je hais les Ecossais. ${ }^{20}$

Pour comprendre l'impact de cette phrase, il faut prendre en compte son contexte socioculturel. Irvine Welsh exploite l'autodérision et la haine de soi du peuple écossais, des sentiments exprimés par un peuple qui se sentait autrefois opprimé. Le livre de Welsh a connu un grand succès au début des années 1990. Le roman a été adapté au théâtre et ensuite au

19. Irvine Welsh, 1993, Trainspotting, London, Minerva Books, p. 76.

20. Eric Lindor Fall, 1996, Trainspotting, Paris, Le Seuil, p. 92 [La traduction commerciale de cet extrait.] 
cinéma. Cette histoire de drogués ne se déroule pas à Glasgow, comme on le croit généralement, mais à Edimbourg. Comme l'annoncent les éditeurs de la version française : 'Fidèle à la langue de la banlieue d'Edimbourg, dont il restitue la gouaille, Trainspotting est un roman à lire à voix haute, avec l'accent'. Certaines phrases du livre, notamment Ah hate the Scots « je hais les Ecossais », et It's shite being Scottish « je suis écossais et c'est merdique » mentionné ailleurs dans le livre ont connu une grande notoriété.

Le paragraphe que nous avons choisi a été repris en entier dans le best-seller du journaliste Jeremy Paxman intitulé The English. L'extrait a également été commenté par le critique Peter Kravitz dans son livre sur la littérature écossaise, qui en fait l'analyse suivante :

«This berating of Scotland from within shows a new self-confidence. Scottish writers are more comfortable criticizing their own country than ever before. This can only come from a degree of cultural security, moving beyond the see-saw of self-love (in the form of blind patriotism) and self-loathing. This was not the case twenty years ago. $»^{21}$

L'extrait a également figuré dans un tract du Scottish National Party, ce qui avait provoqué en 1998 l'intervention de la Commission for Racial Equality, la commission parlementaire veillant au respect de la loi contre les publications racistes. On voit que, si notre auteur n'est pas un Marcel Proust calédonien, il est au moins comparable à Michel Houellebecq, de par le genre de romans qu'il écrit et la polémique que ses textes ont provoquée.

Il y a donc, certainement, originalité du contenu. Mais dans quelle mesure est-ce que notre extrait correspond à ce qui existe déjà en anglais britannique, et dans quelle mesure est-il original du point de vue linguistique? Pour répondre à ces questions, il faut examiner des constructions apparentées à chaque partie de la phrase, que nous divisons en deux.

\section{UNE ENUMERATION D'INVECTIVES}

La première partie de notre expression constitue un groupe nominal (GN) autour de trash pré-modifié par une énumération d'épithètes. Quand on regarde le paragraphe entier, on comprend que cette séquence est la reprise d'une série d'invectives déjà établies dans le paragraphe, What does that make us? The lowest of the fuckin low, the scum of the earth... La présence de l'article peut être simplement expliquée par son emploi obligatoire dans la comparaison superlative The most ... that ever que nous analysons plus loin.

Le lexème trash apparaît 212 fois dans le British National Corpus (BNC). Syntaxiquement, c'est la tête du GN, mais du point de vue expressif ce n'est pas l'élément le plus essentiel : on pourrait même prétendre qu'à la suite d'une telle série d'invectives, ce type de mot est redondant, sinon prévisible. D'ailleurs, cet emploi de trash obéit simultanément à ses catégories d'emplois dans la langue anglaise : trash-I «pourriture », une insulte raciale, et trash-II « foutaise », une œuvre inutile. Parmi les exemples de trash-I on trouve dans le BNC cinq emplois du nominal pré-modifié dans un sens proche de notre exemple, black trash, white trash, bloody IRA trash, en plus d'un nominal pré-modifié par des qualificatifs ungrateful, grasping, foul-minded gutter trash et un nominal pré-modifié par des classificateurs suburban white alcoholic trash. La plupart des exemples expriment ainsi des insultes raciales, ce qui rend cohérent l'emploi de trash par rapport au peuple écossais.

21. Peter Kravitz, 1997, Contemporary Scottish Literature, London, Picador. [Nous proposons la traduction suivante : «Cet auto-dénigrement de l'Ecosse est la preuve d'une nouvelle confiance en soi. Les écrivains écossais critiquent leur propre pays avec une aisance plus désinvolte que jamais. Cela ne peut s'expliquer que par une assurance culturelle qui transcende la vacillation traditionnelle entre l'amour de soi - caractérisé par un patriotisme aveugle - et la haine de soi. Ce n'était pas le cas il y a vingt ans. »] 
Si l'emploi du mot trash précédé par une série de qualificatifs est tout à fait prévisible, qu'en est-il de la construction pathetic trash ? Il n'y a pas d'exemples de pathetic trash dans le BNC, mais une recherche sur Internet (à l'aide du moteur alltheweb.com) révèle 211 occurrences dont approximativement un tiers cite le texte d'Irvine Welsh. Il est intéressant de noter que ces exemples sont souvent partiels, ou comportent des erreurs, ce qui indique que le discours de Trainspotting a été légèrement reformulé et non simplement copié. En voici deux exemples, parmi beaucoup d'autres :

1. The scum of the fucking Earth! The most wretched miserable servile pathetic trash that was ever shat on civilization [Sic].

2. the bleakness of the future, and the humiliation of being Scottish ("the most wretched, servile, miserable, pathetic trash that was ever shagged [Sic] into civilization [Sic]," Renton quips.)

Les autres emplois de pathetic trash que nous trouvons sur Internet (89 exemples) correspondent aux emplois de trash-II, c'est-à-dire des critiques d'émissions, de publications, ou d'autres communications médiatiques, par exemple :

3. If my life was to become so boring as to stoop to watching such pathetic trash I would start campaigning for euthanasia rights!

4. Thank goodness my sisters aren't into that pathetic trash.

5. I can't believe you would use such a technological miracle as the Internet for such pathetic trash!

6. At a time when $98 \%$ of the fiction published right now is pathetic trash, they need to publish some fiction that is well-told, well-written, and thought-provoking.

7. The official documents detailing allegations against Bin Laden can only be described as pathetic trash.

L'emploi de pathetic trash pour insulter des personnes (ressemblant à trash-I) est beaucoup moins fréquent, avec seulement six occurrences sur les milliers de sites accessibles (nous n'en citons que cinq):

8. Would you bottom feeding pieces of pathetic trash shut your $\mathrm{F}^{*} \mathrm{C} *$ ing mouths before I shut each and everyone of them.

9. Eat shit and get lost you pathetic trash

10. My little pork rind snarfers, I've often wondered how you pathetic trash manage to[...]

11. "You bring her back, now, you pathetic trash!!" StarStriker screamed at the empty space.

12. Anyone that insults Ozzy is pathetic trash obviously not worth listening to.

Il est intéressant de noter que ce pathetic trash est la plupart du temps pré-modifié par you. Nous pouvons alors associer à cette expression un contexte rhétorique assez précis : dans le discours familier des internautes anglophones, pathetic trash est une insulte que l'on réserve à un groupe d'interlocuteurs jugés sans valeur. La répétition d'insultes dans cette phrase complète l'accumulation d'invectives dans la première partie du paragraphe.

La construction The most wretched, servile, miserable, pathetic trash représente donc un croisement de deux phraséologies existantes : un juron racial généralement pré-modifié, et une insulte personnelle adressée à des locuteurs identifiés dans un dialogue condescendant. La construction est cohérente parce qu'elle établit à la fois une référence ethnique et une connivence ironique avec les lecteurs.

\section{UNE RESULTATIVE CREATIVE}

Nous passons maintenant à la deuxième partie de notre extrait. Il s'agit d'une relative enchâssée dans une comparaison: The most [...] pathetic trash that was ever shat intae creation. Dans les concordances du BNC, le verbe shit et ses dérivés ne témoignent d'aucune structure de ce type. Certes, on peut l'utiliser dans une construction indirecte, shit on the poor, 
« chier sur les pauvres » et le verbe peut aussi s'employer au réfléchi, to shit oneself, « avoir la trouille, les chocottes ». Mais pour explorer l'originalité véritable de cette phrase, il faut considérer l'imbrication de deux constructions différentes:

a) the most $\mathrm{ADJ}$ GN that was ever VER-ed / the ADJ-est GN that was ever VER-ed.

b) to be VER-ed into creation / to VER GN into creation.

Examinons d'abord la structure comparative (a). Nous pouvons trouver 200 séquences discontinues correspondant au schéma the most ADJ NOM that was ever VER-ed / the ADJest NOM that was ever VER-ed dans le BNC. Les concordances suivantes démontrent qu'il s'agit d'un schéma assez productif, construit généralement autour d'un nominal générique, the most / ADJ-est thing / sight / event... suivi par un procès général that ever happened / took place / occurred. Le verbe le plus fréquent est happen avec 35 occurrences :

13. It is the most awful thing that could ever happen to me.

14. This is the most embarrassing thing that's ever happened to me.

15. it's the most exciting thing which has happened there since the man was buried,

16. But this is the most flattering thing that has ever happened to Howard!

17. And that, I think, is, in humane terms, perhaps the most important thing, that's happening in education today.

On voit dans ces exemples que l'épithète est généralement plus expressif que la tête du groupe nominal. De plus, l'adverbe ever fait souvent partie de ce schéma, où il accentue la particularité de la comparaison. Si le NOM est plus précis, les verbes introduits dans la relative correspondent à un procès existentiel suggérant une apparition, appear, be, befall, come out, exist :

18. Everyone agrees it was the most monstrous wave that had ever appeared on the North Shore.

19. And made him one of the leading authorities on the fourteenth century, which was arguably the most terrible century that had ever been, at least until the present one.

20. We had to avoid vibrations which would turn the beer cloudy potentially the biggest disaster that could befall the town.

21. For all the pay rises in the world, the finest thing that ever came out of the pit was a bath.

22. ...the two great general unions, the T G W U and the G M B, found a way of creating the most powerful union that has ever existed in Britain.

Un petit nombre de verbes expriment des procès mentaux ou communicatifs dans la même construction : hear, know, pronounce, see, write. Ces exemples sont souvent au passif, ce qui rappelle notre exemple :

23. and then embarked on the most elegant, the most pathetic, and the most sublime address that was ever heard, perhaps, within the walls of that building.

24. Together we will form the finest army that Ireland has ever known.

25. Probably the most depressing words that have ever been pronounced about any slimming diet are those enthusiastic phrases from well-meaning medics on the lines of ...

26. I have returned from the toughest trial that I have ever seen.

27. erm it seems to me that they are among the most important, most serious novels that have ever been written anywhere.

Nous trouvons par contre très peu d'exemples exprimant un procès matériel. On remarquera que le NOM est absent :

28. His collections of Birds, quadrupeds, nests and eggs are unquestionably the finest that have ever been brought home.

29. Her island trip that year was to Palros, perhaps the most successful that she ever made. 
30. The action that followed was one of the most bizarre that sailing-ships ever fought.

La construction superlative the most ADJ that (was, has) ever VER signale généralement un événement exceptionnel dont la nature est toujours appréhendée dans la relative. Mais seule la construction dans [28] bring home a collection ressemble à notre exemple, dans la mesure où elle indique un déplacement causé.

Ce dernier exemple nous mène à la structure résultative (b) to be VER-ed into creation qui est comparable au passif to VER GN into creation. Dans les termes que nous avons adoptés plus haut, ces deux constructions sont apparentées à une construction verbale transitive to «atypique » VER GN into creation. Cette structure est parfois qualifiée de biprédication résultative $e^{22}$. Le terme «biprédication » suppose qu'un résultat secondaire implicite dans le prédicat, un déplacement ou un autre changement d'état, serait indépendant du verbe, mais, comme l'explique Claudé pour l'exemple He drank her under the table :

«Il est impossible que l'on 'boive quelqu'un', donc la phrase anglaise 'he drank her under the table' cache des opérateurs ... et on ne peut l'expliciter que par une paraphrase du genre : 'he made her drink, so that she fell under the table'. Le pronom 'her' est COD d'un verbe impliqué dénotant un processus actif, et sujet d'un autre verbe impliqué porteur du sème. ${ }^{23}$

Nous verrons plus loin qu'il n'est pas souhaitable de postuler les termes opérateur et biprédication pour ce type de construction. Pour l'instant, il suffit de constater que l'expression He drank her under the table n'a pas le même sens que la paraphrase proposée par Claudé : He made her drink // so she fell under the table, où c'est he qui cause indirectement le déplacement de she. Nous préférons recourir aux connaissances culturelles pour expliquer notre interprétation d'un tel énoncé. Dans l'esprit des anglophones, cette expression réfère conventionnellement à un événement typiquement associé à un concours de buveurs. Une meilleure reformulation serait plutôt He drank // so she drank herself under the table. Le lien causatif entre les participants et le déplacement causé ne pourrait s'expliquer autrement que par une théorie de référence globale. En outre, le déplacement indiqué par la résultative He drank her under the table n'aurait rien de «biprédicatif »; ce serait plutôt le sens que l'on associe tout à fait conventionnellement à la construction Sujet / Prédicat / Complément direct / Complément indirect (S / P / Cod / Coi $)^{24}$.

L'approche de Goldberg a le mérite de rendre explicite un certain nombre de ressemblances que l'on peut identifier entre des constructions similaires, tout en proposant que les variations de ces séquences témoignent de la «polysémie constructive ». Selon Goldberg, la séquence S / P / Cod / Coi exprime un déplacement abstrait qui n'est pas lié au verbe mais plutôt à la séquence elle-même. Dans les exemples suivants, elle soutient que ni le verbe ni la préposition n'encodent le déplacement, et pourtant nous comprenons qu'il en résulte un mouvement d'une certaine sorte :

\#1 I cannot imagine my way through the dark labyrinth of its distortion.

\#2 They laughed the guy out of town.

\#3 Pat sneezed the napkin off the table.

\#4 Joe squeezed the rubber ball inside the jar.

\#5 Sam urged Bill outside the house.

22. Jacques François, 1989, Changement, causation, action. Trois catégories sémantiques fondamentales du lexique verbal français et allemand. Genève, Droz.

23. Pierre Claudé, 1995, «Vectorialité », Bulletin de l'Association des Professeurs de Langues Vivantes, novembre, No. 51, p. 1.

24. Adele Goldberg, 1995, A Construction Grammar Approach to Argument Structure, Chicago, University of Chicago Press. 
Goldberg conclut que ce ne sont pas les verbes qui déterminent l'argumentation dans ces énoncés, mais la construction (ou séquence) elle-même :

«[the] basic sentences of English are instances of constructions, form-meaning correspondences that exist independently of particular verbs. An entirely lexically-based, or bottom-up, approach fails to account for the full range of English data. Particular semantic structures together with their associated formal expressions must be recognized as constructs independent of the lexical items which instantiate them. ${ }^{25}$

Goldberg suggère ainsi que ce sont les différences de séquence des compléments (directs, indirects et datifs en anglais) qui déterminent les rôles sémantiques autant pour les verbes de mouvement, wander, chase, push, place, send, que pour les verbes qui ne dénotent aucun mouvement, imagine, laugh, sneeze, squeeze, urge. Un verbe qui ne dénote pas directement le sens associé à cette construction signifierait par défaut le moyen par lequel le processus s'effectue. Goldberg applique cette analyse à des séquences considérées synonymiques, notamment S / P / Cod / Coi et S / P / Coi /Cod. Elle refuse de considérer ces séquences comme des paraphrases, voyant à la place des variantes qui peuvent être distinguées par des facteurs contextuels :

«Since we have assumed that no two constructions are entirely synonymous both semantically and pragmatically, it should be possible to find contexts in which a given construction is the most preferred.» ${ }^{26}$

Ainsi, les interprétations des phrases She kicked him the ball et She kicked the ball to him seraient-elles respectivement résultative / directive. De même, une construction ditransitive $\mathrm{S}$ / P / Coi (= Complément datif) / Cod serait réservée aux compléments datifs affectés, She gave the man a kick «Elle a donné un coup de pied à l'homme » ${ }^{27}$. De même, la séquence S / $\mathrm{P} / \mathrm{Cod} /$ Coi serait réservée aux compléments ayant le rôle de destinataire, une interprétation compatible avec un déplacement causé, He whispered the news to the woman «Il murmura les nouvelles à l'oreille de la dame ». Cette différence explique pourquoi la séquence S / P / Cod / Coi ne permet pas d'objet affecté à la place du Coi oblique, *She gave a kick to the man. De même la séquence S / P / Coi / Cod ne permet pas des compléments avec le rôle 'bénéficiaire / destinataire' à la place du datif indirect, *He whispered the women the news. Selon Goldberg, la séquence S / P / Coi / Cod sera toujours interprétée comme une résultative de déplacement.

Cependant, on peut voir dans ces exemples un rôle assez important des verbes dans ces constructions, un fait qui n'est pas commenté par Goldberg. Le fait que Goldberg ne tienne pas compte de la nature lexicale de ces séquences est une conséquence de son approche cognitive, ce qui devient évident quand elle postule que des «structures sémantiques doivent être reconnues indépendamment des occurrences lexicales qui les réalisent » (p.1). Mais il est tout à fait possible de démontrer que les constructions sont liées à un lexique spécifique. Et on pourrait aller plus loin : les constructions elles-mêmes seraient essentiellement de nature lexicale.

Il suffit de regarder de plus près le groupe prépositionnel dans notre exemple to shit someone into creation. Dans le BNC les verbes intransitifs introduisant into creation

25. Adele Goldberg, 1995, A Construction Grammar Approach to Argument Structure, Chicago, University of Chicago Press. p. 1.

26. Adele Goldberg, 1995, ... p. 122.

27. Dans la grammaire de Halliday le complément $a$ kick a le rôle sémantique de portée puisqu'il constitue une expansion nominale du procès matériel et non pas un participant affecté par le procès. 
représentent pour la plupart des procès relationnels où la création est le résultat d'un mouvement non-accusatif, come, go, develop, flow, turn :

31. Thus came into creation a variety of employee benefits not subject to personal income tax.

32. Our philosophy is that music, as an art form, needs a fertile atmosphere to develop fully into creation.

33. Albion in its origin was the Form of forms, the original pattern for all that flowed into creation of the unique and magnificent wonder known as the Celtic spirit.

34. For Kahlo, experience turned into creation, blood into paint, flesh into art.

35. A lot of work went into creation of this Guide, and we hope it is helpful to you.

Ces extraits expriment souvent l'auto-félicitation pour un effort de création particulier. Les verbes de mouvement, develop, flow, turn dénotent tous un changement d'état plutôt qu'un acte de création matérielle.

Une autre catégorie d'emploi est exprimée par le verbe bring, correspondant à la création matérielle au passif comme à l'actif:

36. you have a great idea for a business and, more importantly, the know-how to bring it into creation.

37. The Abyss Replica ring was also first brought into creation by our company, as documented in the commentary in the DVD version of the Abyss movie.

38. The actual act of bringing a child into creation is almost as significant as being there for them, providing for them, and ultimately being their first teacher

39. This recording would not have been possible and this music would not have been brought into creation without the love and support of my family.

40. Man is the central figure; the end of all creation, and the entire workshop of the earth and the skies have been brought into creation solely for him.

Tous ces exemples évoquent un acte de création quasi-biblique, surnaturel ou mystique. Ceci est d'autant plus vrai pour les verbes plus précis que bring exprimant la création mystique par le biais de la voix ou de la communication, breathe, call, sing, speak. De tels exemples sont absents dans le BNC, mais sur Internet on en trouve quatre :

41. Then at a particular time, God had breathed into creation the Spirit, thereby enabling man and woman to know God and communicate with the divine directly.

42. It was certainly the ugliest dog that had ever been called into creation.

43. Aboriginal people talk of 'Creation Songs' - a time when the ancestors were born from the earth and sang the world into creation.

44. The Word made flesh, the Word of God Who spoke the universe into creation. John 1:14

Si la création spontanée ou mystique représente la phraséologie dominante de ces emplois, on trouve, toujours sur Internet, trois exemples de procès matériels, cobble, " concocter », grind, « broyer », spawn, « engendrer», qui référent tous à des processus de création physiquement pénibles :

45. [the time] I have spent in front of my computer cobbling this grammar into creation.

46. His left index finger grounds this duality into creation drawing from the original chaos to bring into being the flowers of creation.

47. Spiked Satanic Rebels Myrkskog was spawned into creation in the year 1993.

C'est dans ces derniers exemples que nous trouvons les emplois les plus proches de notre expression shat into creation, même s'ils sont assez atypiques. Selon le Cobuild, le verbe cobble est plus souvent utilisé avec la particule together pour indiquer la création spontanée. Par contre, grind est plus souvent utilisé avec into mais avec le sens d'enfoncer. De même, l'exemple spawn, « créer, engendrer » est toujours transitif. Voici son entrée dans le dictionnaire : 
Spawn 3. If something spawns something else, it causes it to happen or be created; a literary use. Tyndal's inspired work spawned a whole new branch of science. ${ }^{28}$

Si ces exemples sont particuliers, ils confirment néanmoins le principe lexico-grammatical selon lequel chaque lexème est associé à des constructions régulières, et chaque construction est apparentée à une expression plus ou moins stable. Notre exemple principal et les exemples [36-47] sont tous des variations d'une construction prototypique : S / P / Cod / into creation. Dans tous ces exemples, into creation n'est pas un complément indirect, dans la mesure où il exprime la portée du procès matériel plutôt qu'un participant bénéficiaire ou destinataire, ni un circonstanciel typique, car il exprime un procès central et non périphérique au procès.

Selon notre conception de la lexico-grammaire, une construction ne peut donc être indépendante de l'ensemble des lexèmes employés usuellement dans ces structures, contrairement à ce qu'affirme Goldberg. Les exemples de résultatives intransitives que nous avons identifiées ici sont toujours associés aux verbes de mouvement, come, go, turn. Les expressions transitives sont associées à des procès matériels ou communicatifs, souvent connotant la création mystique, bring, breathe, speak. Les résultatives transitives exprimées au passif sont moins nombreuses, mais expriment sans exception la pénibilité, cobble, grind, spawn. Notre exemple that was ever shat intae creation s'inscrit nettement dans cette dernière phraséologie.

\section{EXPRESSION ET DENOMINATION}

En termes purement descriptifs, la seule originalité dans notre exemple The most wretched, servile, miserable, pathetic trash that was ever shat intae creation est la collocation du verbe shit avec la préposition into. Mais après une analyse plus fine des corpus disponibles, la véritable originalité d'Irvine Welsh s'avère être la juxtaposition de deux constructions déjà productives de l'anglais, notamment une comparaison relative, associée à l'accumulation d'injures raciales, the most ADJ (+ADJ +ADJ...) NOM that ... et une résultative au passif, associée à un acte de création corporelle, ... that was ever VER-ed into creation. En fin de compte, l'emploi apparemment paradoxal du verbe shit devient tout à fait cohérent dans un contexte où le produit de la création, le peuple écossais, est présenté métaphoriquement comme une ordure, trash.

On pourrait donc supposer que l'innovation de Welsh n'est rien d'autre que la conjonction nouvelle de quelques éléments linguistiques, la création d'une nouvelle collocation. Mais l'impact de cette phrase dépend également de son emplacement à la fin d'un paragraphe qui nous mène, par son rythme et sa séquence d'invectives, à une paroxysme sciemment choquant. La créativité stylistique de Welsh dépend ainsi de facteurs culturels, sociaux et textuels.

Pourtant, ce qui nous concerne ici, c'est l'émergence d'une nouvelle phraséologie. Pour traiter cette question, on ne peut se contenter d'une approche descriptive qui identifie seulement les originalités formelles de l'énoncé. Il faut prendre en compte, comme nous l'avons souligné plus haut, le passage d'une série de constructions à une expression entière. Pour nous, le terme construction réfère à des correspondances lexico-grammaticales que l'on peut identifier dans un extrait de ce type et que l'on peut confronter à la phraséologie ambiante de l'anglais britannique. Mais selon le principe lexico-grammatical de Halliday, les constructions ne permettent de faire que la moitié du travail : pour comprendre l'impact de cet extrait et sa portée socioculturelle, il est nécessaire de considérer la valeur de l'ensemble, notamment son existence potentielle en tant que texte unique et dissociable. Notre terme expression évoque plutôt la portabilité ou la qualité transférable d'une phrase qui est devenue emblématique. L'extrait dont nous parlons est très connu dans le contexte écossais, et s'il a

28. John Sinclair (réd), 1995, Collins COBUILD English Dictionary, London, Harper Collins, p. 1600. 
fait partie du discours politique de l'Ecosse pendant un certain temps, c'est qu'il a en quelque sorte représenté l'ouvrage tout entier, qui apportait quelque chose de valorisant à la production culturelle écossaise, même s'il était extrêmement critique des mœurs du pays. Irvine Welsh a ainsi forgé une sorte de néologisme textuel qui a été repris par sa communauté. C'est pour cette raison que nous insistons sur la distinction entre construction (obéissant aux contraintes de la lexico-grammaire) et expression (une construction dont la reconnaissance et l'interprétation dépendent de l'usage). Tandis que les constructions doivent se formuler dans les limites d'une grammaire de l'énoncé, que ce soient des monèmes, des synthèmes ou des phrasèmes plus complexes, les expressions sont des entités référentielles. Les expressions ne sont pas définies en effet par leur structure ou leur sémantisme, qui peuvent être réduites à des facteurs compositionnels, mais plutôt par leur capacité à référer. C'est pour cette raison que notre extrait peut servir d'emblème pour le paragraphe ou même l'ouvrage entier.

En effet, le moteur de la créativité est la référence, c'est-à-dire notre capacité à utiliser des signes publics pour donner une forme à une pensée qui sans les signes ne serait qu'un magma évanescent. C'est ce qu'Irvine Welsh a fait en parlant ainsi de l'identité écossaise. Il a ensuite eu la chance de voir un fragment de son texte repris par sa communauté et réutilisé, même approximativement, par de nombreux locuteurs pour leur propre compte (les exemples [1-2] cités plus haut). C'est sans doute le destin de toute expression néologique quand elle est acceptée. Mais ce n'est pas le seul critère. Nous supposons aussi que le néologisme se fait uniquement lorsqu'il devient évident pour tous que cette expression dénomme (nomme, ou réfère à) un nouvel objet social. L'expression n'existe donc pas si elle ne remplit pas les critères sémiotiques de la dénomination, un terme issu de la tradition médiévale pour signaler une classe d'expressions qui i) sont reconnues par la communauté linguistique, et qui ii) réfèrent à une catégorie stable de notre expérience.

Nos termes construction, expression, dénomination ne constituent pas une hiérarchie formelle, qui serait plutôt désignée par des termes comme collocation, colligation, synthème et phrasème dont nous avons discuté plus haut. Il s'agit plutôt d'une série de perspectives. Les séquences simples comme strong tea et faire la sieste, ou plus complexes, comme She laughed her way through the crowd sont des constructions du point de vue lexicogrammatical, mais aussi des expressions selon les critères sémiotiques. Si nous désirons séparer les constructions différentes, nous pouvons nous référer comme le font Martinet et Depecker, à leur degré de détermination : les synthèmes seraient plus incomplets, des constructions minimales dont les éléments respectifs ne sont pas déterminables individuellement, tandis que les phrasèmes seraient des assemblages de constructions. Mais ce qui importe pour nous est que ces unités désignent globalement un objet de notre expérience, quelle que soit leur taille, leur figement syntaxique ou leur degré d'opacité sémantique. La dénomination désigne plutôt la relation que l'on peut établir entre une expression (une forme portable, que ce soit un mot ou un texte entier) et une catégorie (un objet sémiotique ou un signe complexe).

Cette approche nous permet d'aborder le problème de la collocation, notamment la distinction épineuse entre collocation et combinaison libre. Nous avons vu que le terme construction rend ces termes désuets, dans la mesure où toute construction, même «inédite ", doit obéir aux contraintes du système lexico-grammatical. Nous soutenons que le figement lexical que l'on peut observer ne provient pas d'une 'colle' qui relie les éléments différents de la collocation, mais de l'habitude que nous avons de les utiliser ensemble pour référer à tel ou tel objet. Le sens de strong tea ou de faire une sieste ne provient pas de la composition de leurs parties, pas plus que l'usage d'une unité monolexicale comme psychothérapeute n'est déterminé par la somme des monèmes qui la composent. Ces parties sont seulement des indices des traces de l'effort néologique qui a été fait au moment de la création de la construction. La capacité des entités lexicales à référer est le fruit d'une habitude : nous avons 
l'habitude d'utiliser les mots de telle ou telle façon, et cela ne nécessite pas d'explications platoniciennes en termes de concepts, d'opérateurs, de relations, ou d'autres termes relationnels qui, comme biprédication résultative, ne font que nommer le mystère sans l'expliquer. Pas plus d'ailleurs que notre capacité à lacer nos souliers ne nécessite d'explications en termes de concepts de lacets, de chaussures, de nœuds et de papillons. Nous savons utiliser les mots, comme nous savons marcher ou lacer nos chaussures. En utilisant l'expression anglaise strong tea, nous ne créons pas du discours neuf et original, nous réemployons cette unité pour désigner la concentration relative d'une substance (on parle ainsi de strong wine, strong medicine). Et si nous pouvons préciser cet attribut, c'est que le thé pourrait aussi bien ne pas le posséder, ou pas de manière absolue. C'est pourquoi nous pouvons aussi utiliser weak, ordinary, rich, robust ou watery à la place de strong. Cette expression correspond bien sûr à une construction nominale semi-figée (un synthème), reconnue et donc distinguée des autres ensembles possibles, comme *powerful tea, que l'on ne dit pas. Seul strong tea est habilité, par convention et non par le sémantisme de ses parties, à référer à une boisson du point de vue de sa concentration en tanins et en théine.

Une expression (du point de vue lexicologique) ou une dénomination (du point de vue sémiotique) est une entité lexicale qui désigne une classe ouverte d'objets, et cette capacité à référer ne dépend pas de ses composants, qui peuvent avoir été oubliés, comme dans au fur et à mesure. Si on admettait que le sens du tout est le résultat de la composition des parties, alors le linguiste serait amené à accepter des hypothèses ontologiques extrêmement fortes (et sans doute hasardeuses) sur l'existence de primitives, sur une langue comme miroir de la pensée et la parole comme construction régulée. Or ces points de vue, même s'ils semblent aller de soi pour la plupart, ont été vivement discutés par des auteurs comme L. Wittgenstein ${ }^{29}$, Ch. Peirce $^{30}$, et M. Merleau-Ponty ${ }^{31}$, sur lesquels nous nous appuyons pour l'argumentation de ce texte. Nous n'avons malheureusement pas la place de présenter ici, ni l'argument du langage privé de Wittgenstein, ni la notion de signe interprétant de Peirce, ni l'affirmation de MerleauPonty que la pensée n'est rien d'intérieur. Le lecteur intéressé pourra se reporter à Frath ${ }^{32}$, pour une analyse et une synthèse de ces points de vue.

On pourrait qualifier notre perspective de «nominaliste ». Pour revenir à l'exemple de Welsh, nous ne considérons pas cet extrait comme une représentation d'un état de faits déjà existant, ou le résultat d'un contexte donné : il s'agit plus simplement d'une création textuelle qui n'a de sens que dans un contexte très précis et, d'ailleurs, très éphémère. Irvine Welsh ne s'est pas amusé à concocter une expression originale gratuitement. Il n'a pas non plus formulé d'abord la pensée que les Ecossais ont été créés par l'activité excrémentielle de quelque entité non spécifiée, une pensée qu'il aurait ensuite traduite en anglais. Il a plutôt fait usage de signes publics disponibles dans sa langue, qu'il a agencés d'une certaine manière au fil de la plume, donnant ainsi une dénomination à un nouvel objet social, celui d'une certaine autodérision des Ecossais affirmant ainsi, paradoxalement, une nouvelle assurance quant à leur identité culturelle. S'il avait écrit ce texte dans une autre langue, il aurait fait usage d'autres signes publics et l'expression aurait été différente. Ainsi, il semble difficile d'imaginer un équivalent en français de shit into creation, cette langue ne disposant pas de la construction résultative. Mais nul doute qu'un écrivain de qualité dans une autre langue ne

29. Ludwig Wittgenstein, 1961, Tractatus logico-philosophicus suivi de Investigations philosophiques, traduit par P. Klossowski, Paris, Gallimard, 1965 [1re édition 1958, Londres, Basil Blackwell], Le cahier bleu et le cahier brun, Paris, Gallimard, 1963, [1re édition 1953, Londres, Basil Blackwell], Philosophical Investigations, traduit par G.E.M. Anscombe, Londres, Basil Blackwell.

30. Charles S. Pierce, 1978, Ecrits sur le signe, rassemblés, traduits et commentés par Gérard Deledalle, Paris, Seuil.

31. Maurice Merleau-Ponty, 1945, Phénoménologie de la perception, Paris, Gallimard.

32. Pierre Frath, à paraître, Signe, référence et usage, Editions Le Manuscrit. 
réussisse à produire une expression tout aussi frappante en faisant usage des dénominations disponibles. 